\title{
Surfaces of finite III-type
}

\author{
Hassan Al-Zoubi \\ Al-Zaytoonah University of Jordan \\ P.O. Box 130, 11733 \\ Amman Jordan
}

Received: July 28, 2021. Revised: October 15, 2021. Accepted: November 3, 2021.

Published: December 3, 2021.

\begin{abstract}
In this paper, we consider surfaces of revolution in the 3 -dimensional Euclidean space $E^{3}$ with nonvanishing Gauss curvature. We introduce the finite Chen type surfaces with respect to the third fundamental form of the surface. We present a special case of this family of surfaces of revolution in $E^{3}$, namely, surfaces of revolution with $R$ is constant, where $R$ denotes the sum of the radii of the principal curvature of a surface.
\end{abstract}

Keywords - Surfaces in the Euclidean 3-space, Surfaces of finite Chen-type, Beltrami operator, Surfaces of revolution.

\section{INTRODUCTION}

$\mathrm{O}$ $\mathrm{NE}$ of the most interesting and profound aspects of differential geometry is the idea of surfaces of finite type which was born by B-Y. Chen in the early 1970s and since then, it has become a source of interest for many researchers in this field. The reader can refer to [10] for more details. In the framework of this kind of study the first-named author with S. Stamatakis have given in [18] a new generalization to this area of study by giving a similar definition of surfaces of finite type.

Let $\boldsymbol{r}$ be an isometric immersion of a surface $S$ in the 3dimensional Euclidean space $E^{3}$. We represent by $\Delta^{J}$ the Laplacian operator of $S$ acting on the space of smooth functions $\mathrm{C}^{\infty}(S)$. Then $S$ is said to be of finite $J$-type, $J=I, I I$, $I I I$, if the position vector $\boldsymbol{r}$ of $S$ can be decomposed as a finite sum of eigenvectors of $\Delta^{J}$ of $S$, that is

$$
\boldsymbol{r}=\boldsymbol{c}+\sum_{i=1}^{k} \boldsymbol{x}_{i},
$$

where

$$
\Delta^{J} \boldsymbol{x}_{\mathrm{i}}=\lambda_{i} \boldsymbol{x}_{i}, i=1, \ldots, k,
$$

$\boldsymbol{c}$ is a fixed vector and $\lambda_{1}, \lambda_{2}, \ldots, \lambda_{k}$ are eigenvalues of the operator $\Delta^{J}$.

Surfaces of finite type according to the second or third fundamental forms became a topic of active research in the last years. Surfaces of finite type in $E^{3}$ regarding to the second fundamental form were investigated for some important classes of surfaces. More precisely, the class of ruled surfaces where studied in [7], while in [3], H. AL-Zoubi studied tubes in $E^{3}$. Other classes such as translation surfaces, Quadric surfaces, surfaces of revolution, helicoidal surfaces, cyclides of Dupin and spiral surfaces, the classification of its finite $I I$ type surfaces still unknown. According to the third fundamental form, ruled surfaces in [4], tubes in [5] and quadric surfaces are the only classes were investigated in $E^{3}$.

This type of study can be also extended to any smooth map, not necessary for the position vector of the surface, for example, the Gauss map of a surface. Regarding this see [8].

Another generalization to the above, one can study surfaces in $E^{3}$ whose position vector $\boldsymbol{r}$ satisfies the following condition

$$
\Delta^{J} \boldsymbol{r}=\mathrm{A} \boldsymbol{r}, \quad J=I I, I I I,
$$

where $\mathrm{A} \in \mathfrak{R}^{3 \times 3}$.

Related to this, in [19] it was proved that the spheres and the catenoids are the only surfaces of revolution satisfying the above equation. Similarly, in [1] it was shown that, helicoids and spheres are the only quadric surfaces in $E^{3}$ that satisfy (2). Next, in [2] condition (2) was studied for the class of translation surfaces. In fact authored ascertained that Scherk's surface is the only translation surface in the Euclidean 3-space that satisfies (2), finally, in [17] the authors studied the class of translation surfaces in $\mathrm{Sol}_{3}$ satisfying (2). Surfaces satisfying condition (2) are said to be of coordinate finite $J$-type [13].

In order to achieve our goal, we briefly introduce a formula for $\Delta^{I I I} \boldsymbol{r}$ and $\Delta^{I I I} \boldsymbol{N}$ by using tensors calculations, where $\boldsymbol{N}$ denotes the normal vector field of $S$. Further, in the last section, we contribute to the solution of our main result.

\section{BASIC CONCEPTS}

Let $S$ be a smooth surface in $E^{3}$ given by a patch $\boldsymbol{r}=\boldsymbol{r}\left(u^{1}, u^{2}\right)$ on a region $\mathrm{U}:=(a, b) \times \Re$ of $\mathfrak{R}^{2}$ in which does not contain parabolic points. We denote by

$$
I=g_{i j} d u^{i} d u^{j}, \quad I I=b_{i j} d u^{i} d u^{j}, \quad I I I=e_{i j} d u^{i} d u^{j}
$$

the first, second and third fundamental forms of $S$ respectively. For two sufficiently differentiable functions $f\left(u^{1}, u^{2}\right)$ and $g\left(u^{1}, u^{2}\right)$ on $S$, the first differential parameter of Beltrami with 
respect to the third fundamental form $J$ is defined by [15]

$$
\nabla^{J}(f, g):=d^{i j} f_{/ i} g_{/ j}
$$

where $f_{i i}:=\frac{\partial f}{\partial u^{i}}$ and $\left(d^{i j}\right)$ denotes the inverse tensor of $\left(g_{i j}\right)$, $\left(b_{i j}\right)$ and $\left(e_{i j}\right)$ for $J=I, I I$ and $I I I$ respectively.

The second differential parameter of Beltrami with respect to the fundamental form $J=I, I I, I I I$ of $S$ is defined by [10]

$$
\Delta^{J} f:=-d^{i j} \nabla_{i}^{J} f_{/ j}
$$

where $f$ is sufficiently differentiable function, $\nabla_{i}^{J}$ is the covariant derivative in the $u^{i}$ direction with respect to the fundamental form $J$ [7].

If we apply (3) for the position vector $\boldsymbol{r}$ with respect to the fundamental form $I I I$, it can be easily verified that [18]

$$
\Delta^{I I I} \boldsymbol{r}=\operatorname{grad}^{I I I} R-R N .
$$

By applying (3) for the normal vector $N$ we get [18]

$$
\Delta^{I I I} N=2 N
$$

From (5) we find that the Gauss map of every surface $S$ in $E^{3}$ is of finite $I I I$-type 1 with eigenvalue $\lambda=2$ [18].

Let $S^{*}$ be a parallel surface of $S$ in (directed) distance $\mu=$ const. $\neq 0$, so that $1-2 \mu H+\mu^{2} K \neq 0$, where $K$ is the Gauss curvature and $H$ is the mean curvature of $S$ respectively. Then $S^{*}$ possesses the position vector $\boldsymbol{r}^{*}=\boldsymbol{r}+\mu \boldsymbol{N}$.

Denoting by $K^{*}$ and $H^{*}$ the Gauss and mean curvature of $S^{*}$ respectively, we mention the following relations [15]

$$
\begin{aligned}
K^{*} & =\frac{K}{1-2 \mu H+\mu^{2} K}, \\
H^{*} & =\frac{H-\mu K}{1-2 \mu H+\mu^{2} K} .
\end{aligned}
$$

Hence we get

$$
R^{*}=\frac{2 H^{*}}{K^{*}} \cdot=R-2 \mu
$$

On the other hand, the surfaces $S, S^{*}$ have common unit normal vector and spherical image. Thus $I I I=I I I^{*}$ and $\Delta^{I I I}=$ $\Delta^{I I I^{*}}$. We prove now the following theorem for later use.

Theorem 1. Let $S$ be a minimal surface in $E^{3}$. Then $S^{*}$ is parallel surface of $S$ if and only if the sum of the principal radii of curvature $R^{*}$ of $S^{*}$ is constant.

Proof. Suppose that $S$ is minimal surface in $E^{3}$, which is defined on a simply connected domain $D$ in the $\left(u^{1}, u^{2}\right)$-plane. Let

$$
S^{*}: r^{*}=r+\mu N, \mu \neq 0
$$

be parallel surface of $S$.

From (6) and taking into account $H=0$, we find $R^{*}=-2 \mu=$ const.. Hence the first part of the theorem is proved.
Conversely, let $R^{*}=$ const. $\neq 0$. Then from Theorem (4.4) (see [18]), $S^{*}$ is of null $I I I$ - type 2 . Therefore from (1) there exist nonconstant eigenvectors $\boldsymbol{x}_{1}\left(u^{1}, u^{2}\right)$ and $\boldsymbol{x}_{2}\left(u^{1}, u^{2}\right)$ defined on the same domain $D$ such that

$$
r^{*}=x_{1}+x_{2}
$$

where $\Delta^{I I I} \boldsymbol{x}_{1}=\lambda_{1} \boldsymbol{x}_{1}, \Delta^{I I I} \boldsymbol{x}_{2}=\lambda_{2} \boldsymbol{x}_{2}$, and here we have $\lambda_{1}=0$ because $S^{*}$ is of null III- type 2 .

Once we have $\Delta^{I I I} \boldsymbol{r}^{*}=\Delta^{I I I} \boldsymbol{x}_{1}+\Delta^{I I I} \boldsymbol{x}_{2}$, it then follows that

$$
\Delta^{I I I} \boldsymbol{r}^{*}=\lambda_{2} \boldsymbol{x}_{2}
$$

On the other hand, since $R^{*}=$ const. $\neq 0$, we get

$$
\Delta^{I I I} \boldsymbol{r}^{*}=-R^{*} N
$$

Thus from (8) and (9), one finds

$$
\lambda_{2} x_{2}=-R^{*} N
$$

or $\boldsymbol{x}_{2}=c N$, where $\mathrm{c}=-\frac{R^{*}}{\lambda_{2}}$, and then (7) becomes

$$
r^{*}=x_{1}+c N
$$

The differential of the above equation is

$$
d \boldsymbol{r}^{*}=d \boldsymbol{x}_{1}+c d N
$$

Taking the inner product of both sides of (11) with $N$ yields

$$
\left\langle d \boldsymbol{x}_{1}, \boldsymbol{N}>=0\right.
$$

Now we want to show that $\boldsymbol{x}_{1}\left(u^{1}, u^{2}\right)$ is a regular parametric representation of a surface in $E^{3}$. It is enough to prove that

$$
\boldsymbol{x}_{1 / 1} \times \boldsymbol{x}_{1 / 2} \neq \mathbf{0}, \quad \forall\left(u^{1}, u^{2}\right) \in D,
$$

where $\times$ is the Euclidean cross product. We have

$$
x_{1}=r^{*}-\mu N^{*}
$$

Using the Wingarten equations [15]

$$
N_{/ i}=-b_{i j} g^{j k} \boldsymbol{r}_{/ k}^{*}
$$

and the equation (13), it follows that

$$
\begin{gathered}
\boldsymbol{x}_{1 / 1} \times \boldsymbol{x}_{1 / 2}=\left(\boldsymbol{r}_{/ 1}^{*}-\mu \boldsymbol{N}_{/ 1}\right) \times\left(\boldsymbol{r}_{/ 2}^{*}-\mu \boldsymbol{N}_{/ 2}\right) \\
=\left(\boldsymbol{r}_{/ 1}^{*} \times \boldsymbol{r}_{/ 2}^{*}\right)-\mu\left(\boldsymbol{r}_{/ 1}^{*} \times \boldsymbol{N}_{/ 2}\right)+\mu\left(\boldsymbol{r}_{/ 2}^{*} \times \boldsymbol{N}_{/ 1}\right)+\mu^{2}\left(\boldsymbol{N}_{/ 1} \times \boldsymbol{N}_{/ 2}\right) \\
=\left(1-2 \mu H+\mu^{2} K\right)\left(\boldsymbol{r}_{/ 1}^{*} \times \boldsymbol{r}_{/ 2}^{*}\right) \neq \mathbf{0}, \quad \forall\left(u^{1}, u^{2}\right) \in D .
\end{gathered}
$$

Hence, on account of (12) and (14), we conclude that $\boldsymbol{x}_{1}\left(u^{1}\right.$, $u^{2}$ ) is a regular parametric representation of a surface in $E^{3}$ with $\boldsymbol{N}$ it's Gauss map. 
Since $\Delta^{I I I} \boldsymbol{x}_{1}=\mathbf{0}$. Consequently, from Theorem (3.1) (see [18]), $\boldsymbol{x}_{1}\left(u^{1}, u^{2}\right)$ is a minimal surface. Thus from (10) we obtain that $S^{*}$ is parallel surface of a minimal. Now we mention and prove our main theorem.

Theorem 2. The only surfaces of revolution in $E^{3}$ of which the sum of the radii of the principal curvature $R$ is constant are

- parts of spheres which are of finite III-type 1,

- catenoids which are of finite null III-type 1, and

- the parallel surfaces to the catenoids, which are of finite null III-type 2.

\section{PROOF OF THEOREM 2}

Let $C$ be a smooth curve lies on the $x z$-plane parametrized by

$$
\boldsymbol{x}(u)=(f(u), 0, g(u)), u \in J,(J \subset \Re),
$$

where $f, g$ are smooth functions and $f$ is a positive function. When $C$ is revolved about the $z$-axis, the resulting point set $S$ is called the surface of revolution generated by the curve $C$. In this case, the $z$-axis is called the axis of revolution of $S$ and $C$ is called the profile curve of $S$. On the other hand, a subgroup of the rotation group which fixes the vector $(0,0,1)$ is generated by

$$
\left(\begin{array}{ccc}
\cos v & -\sin v & 0 \\
\sin v & \cos v & 0 \\
0 & 0 & 1
\end{array}\right)
$$

Then the position vector of $S$ is given by

$$
\begin{gathered}
\boldsymbol{r}(u, v)=(f(u) \cos v, f(\mathrm{u}) \sin v, g(u)), \\
u \in J, v \in[0,2 \pi) .
\end{gathered}
$$

(For the parametric representation of surfaces of revolution, see $[14,16])$.

Without loss of generality, we may assume that $C$ has the arc-length parametrization, i.e., it satisfies

$$
\left(f^{\prime}\right)^{2}+\left(g^{\prime}\right)^{2}=1
$$

where

$':=\frac{d}{d u}$. Furthermore if $f^{\prime} g^{\prime}=0$, then $f=$ const. or $g=$ const. and $S$ would be a circular cylinder or part of a plane, respectively. A case which has been excluded since $S$ would consist only of parabolic points [19].respectively. Hence $S$ would consist only of parabolic points, which has been excluded.

The partial derivatives of (15) are

and

$$
\boldsymbol{r}_{u}=\left(f^{\prime}(u) \cos v, f^{\prime}(u) \sin v, g^{\prime}(u)\right)
$$

$$
\boldsymbol{r}_{v}=(-f(u) \sin v, f(u) \cos v, 0) .
$$

The components $g_{i j}$ of the first fundamental form in (local) coordinates are the following

$$
g_{11}=\left\langle\boldsymbol{r}_{u}, \boldsymbol{r}_{u}\right\rangle=1, g_{12}=\left\langle\boldsymbol{r}_{\mathrm{u}}, \boldsymbol{r}_{v}\right\rangle=0, g_{22}=\left\langle\boldsymbol{r}_{v}, \boldsymbol{r}_{v}\right\rangle=f^{2}
$$

Denoting by $\kappa$ the curvature of the curve $C$ and $R_{1}, R_{2}$ the principal radii of curvature of $S$, we have

$$
R_{1}=\frac{1}{\kappa}, \quad R_{2}=\frac{f}{g^{\prime}},
$$

The Gauss curvature and the mean curvature of $S$ are respectively

$$
\begin{gathered}
K=\frac{1}{R_{1} R_{2}}=\frac{\kappa g^{\prime}}{f}=-\frac{f^{\prime \prime}}{f}, \\
2 H=\frac{1}{R_{1}}+\frac{1}{R_{2}}=\kappa+\frac{g^{\prime}}{f} .
\end{gathered}
$$

The Gauss map $N$ of $S$ is computed as follows

$$
N(u, v)=\left(-g^{\prime} \cos v,-g^{\prime} \sin v,-f^{\prime}\right) .
$$

Now, by using the natural frame $\left\{\boldsymbol{N}_{u}, \boldsymbol{N}_{v}\right\}$ of $S$ defined by

and

$$
\boldsymbol{N}_{u}=\left(-g^{\prime \prime} \cos v,-g^{\prime \prime} \sin v, f^{\prime \prime}\right)
$$

$$
\boldsymbol{N}_{v}=\left(g^{\prime} \sin v,-g^{\prime} \cos v, 0\right)
$$

the components $e_{i j}$ of the third fundamental form in (local) coordinates are the following

$$
\begin{gathered}
e_{11}=<\boldsymbol{N}_{u}, \boldsymbol{N}_{u}>=\left(g^{\prime \prime}\right)^{2}+\left(f^{\prime \prime}\right)^{2}, \\
e_{12}=<\boldsymbol{N}_{u}, \boldsymbol{N}_{v}>=0, \mathrm{e}_{22}=<\boldsymbol{N}_{v}, \boldsymbol{N}_{v}>=\left(g^{\prime}\right)^{2} .
\end{gathered}
$$

The Beltrami operator $\Delta^{I I I}$ in terms of local coordinates $(u$, $v$ ) of $S$ can be expressed as follows

$$
\Delta^{I I I}=-\frac{1}{\kappa^{2}} \frac{\partial^{2}}{\partial u^{2}}+\frac{g^{\prime} \kappa^{\prime}-\kappa g^{\prime \prime}}{g^{\prime} \kappa^{3}} \frac{\partial}{\partial u}-\frac{1}{g^{\prime 2}} \frac{\partial^{2}}{\partial v^{2}} .
$$

On account of (16) we put

$$
f^{\prime}=\cos \varphi, g^{\prime}=\sin \varphi,
$$

where $\varphi=\varphi(u)$. Then $\kappa=\varphi^{\prime}$ and the parametric representation (17) of the unit vector $N$ of $S$ becomes

$$
N(u, v)=\{-\sin \varphi \cos v,-\sin \varphi \sin v, \cos \varphi\} .
$$

Also relation (18) takes the following form

$$
\Delta^{I I I}=-\frac{1}{\varphi^{\prime 2}} \frac{\partial^{2}}{\partial u^{2}}+\left(\frac{\varphi^{\prime \prime}}{\varphi^{\prime 3}}-\frac{\cos \varphi}{\varphi^{\prime} \sin \varphi}\right) \frac{\partial}{\partial u}-\frac{1}{\sin ^{2} \varphi} \frac{\partial^{2}}{\partial v^{2}} .
$$


For the sum of the principal radii of curvature $R=R_{1}+R_{2}$ $=\frac{2 H}{K}$, one finds

$$
R=\frac{f}{\sin \varphi}+\frac{1}{\varphi^{\prime}} .
$$

Taking the derivative of (21) we find

$$
R^{\prime}=-\frac{\varphi^{\prime \prime}}{\varphi^{\prime 2}}-\frac{f \varphi^{\prime} \cos \varphi}{\sin ^{2} \varphi}+\frac{\cos \varphi}{\sin \varphi}
$$

Let $\left(r_{1}, r_{2}, r_{3}\right)$ be the coordinate functions of (15). By virtue of (20) we obtain

$$
\begin{gathered}
\Delta^{I I I} r_{1}=\Delta^{I I I}(f \cos v)= \\
\left(\frac{\varphi^{\prime \prime} \cos \varphi}{\varphi^{\prime 3}}-\frac{1}{\varphi^{\prime} \sin \varphi}+\frac{2 \sin \varphi}{\varphi^{\prime}}+\frac{f}{\sin ^{2} \varphi}\right) \cos v \\
\Delta^{I I I} r_{2}=\Delta^{I I I}(f \sin v)= \\
\left(\frac{\varphi^{\prime \prime} \cos \varphi}{\varphi^{\prime 3}}-\frac{1}{\varphi^{\prime} \sin \varphi}+\frac{2 \sin \varphi}{\varphi^{\prime}}+\frac{f}{\sin ^{2} \varphi}\right) \sin v \\
\Delta^{I I I} r_{3}=\Delta^{I I I} \mathrm{~g}=-\frac{2 \cos \varphi}{\varphi^{\prime}}+\frac{\varphi^{\prime \prime} \sin \varphi}{\varphi^{\prime 3}} .
\end{gathered}
$$

From (21) and (22), equations (23), (24) and (25) become respectively

$$
\begin{aligned}
& \Delta^{I I I} r_{1}=\left(R \sin \varphi-\frac{R^{\prime} \cos \varphi}{\varphi^{\prime}}\right) \cos \nu, \\
& \Delta^{I I I} r_{2}=\left(R \sin \varphi-\frac{R^{\prime} \cos \varphi}{\varphi^{\prime}}\right) \sin \nu, \\
& \Delta^{I I I} r_{3}=-\frac{R^{\prime} \sin \varphi}{\varphi^{\prime}}-R \cos \varphi .
\end{aligned}
$$

We obtain the following two cases:

Case $I . R \equiv 0$.

Thus $H \equiv 0$. Consequently $S$, being a minimal surface of revolution, is a catenoid [19].

Case II. $R=$ const. $\neq 0$.

From (26), (27) and (28) we obtain

$$
\left.\begin{array}{c}
\Delta^{I I I} r_{1}=R \sin \varphi \cos v \\
\Delta^{I I I} r_{2}=R \sin \varphi \sin v \\
\Delta^{I I I} r_{3}=-R \cos \varphi
\end{array}\right\}
$$

Let $\left(N_{1}, N_{2}, N_{3}\right)$ be the coordinate functions of $N$. From (18), relations (29) can be written

$$
\Delta^{I I I} r_{1}=-R N_{1}, \Delta^{I I I} r_{2}=-R N_{2}, \Delta^{I I I} e_{3}=-R N_{3},
$$

and hence

$$
\Delta^{I I I} \boldsymbol{r}=-R N .
$$

In view of (5) and (30) we have

$$
\left(\Delta^{I I I}\right)^{m} \boldsymbol{r}=-\left(2^{m-1}\right) R \boldsymbol{N} .
$$

Now, if $S$ is of finite type $k$, then there exist real numbers $c_{1}$, $c_{2}, \ldots, c_{k}$ such that

$$
\left(\Delta^{I I I}\right)^{k} \boldsymbol{r}+c_{1}\left(\Delta^{I I I}\right)^{k-1} \boldsymbol{r}+\ldots+c_{k} \boldsymbol{r}=\mathbf{0} .
$$

From (30) and (31) relation (32) becomes

$$
\begin{gathered}
-2^{\mathrm{k}-1} R \boldsymbol{N}-2^{\mathrm{k}-2} c_{l} R \boldsymbol{N}-\ldots-c_{k-1} R \boldsymbol{N}+c_{k} \boldsymbol{r}=\mathbf{0}, \\
\text { or } \quad c \boldsymbol{N}+c_{k} \boldsymbol{r}=\mathbf{0},
\end{gathered}
$$

where $\mathrm{c}=-R\left(2^{\mathrm{k}-1}+2^{\mathrm{k}-2} c_{1}+\ldots+c_{k-1}\right)=$ const.

Now, if $c_{\kappa} \neq 0$, then from (33) we have $\boldsymbol{r}=-\frac{c}{c_{k}} N$, and hence we get $|r|=\left|\frac{c}{c_{k}}\right|$ and so $S$ is a sphere. On account of Theorem (3.3) (see [18]), $S$ is of finite $I I I$-type 1 . If $c_{k}=0$, then $S$ is of null type k. Since $R=$ const., thus according to Theorem (4.4) (see [18]) and Theorem (1), $S$ is of null III-type 2 which is parallel surface of a minimal.

\section{REFERENCES}

[1] H. Al-Zoubi, S. Stamatakis, Ruled and Quadric surfaces satisfying $\Delta^{I I I} \boldsymbol{x}=A \boldsymbol{x}$, J. for Geom. and Graphics, 20 (2016), pp.147-157.

[2] H. Al-Zoubi, S. Stamatakis, W. Al Mashaleh and M. Awadallah, Translation surfaces of coordinate finite type, Indian J. of Math. 59 (2017), pp. 227-241.

[3] H. Al-Zoubi, Tubes of finite II-type in the Euclidean 3space, WSEÁS Trans. Math. 17 (2018), pp. 1-5.

[4] H. Al-Zoubi, S. Al-Zubi, S. Stamatakis and H. Almimi, Ruled surfaces of finite Chen-type. J. Geom. and Graphics 22 (2018), pp. 15-20.

[5] H. Al-Zoubi, K. M. Jaber, S. Stamatakis, Tubes of finite Chen-type, Commun. Korean Math. Soc. 33 (2018), pp. 581-590.

[6] H. Al-Zoubi, M. Al-Sabbagh, S. Stamatakis, On surfaces of finite Chen III-type, Bull. Belgian Math. Soc. 26 (2019), pp. 177-187.

[7] H. Al-Zoubi, A. Dababneh, M. Al-Sabbagh, Ruled surfaces of finite II-type, WSEAS Trans. Math. 18 (2019), pp. 1-5.

[8] H. Al-Zoubi, T. Hamadneh, M. Abu Hammad, and M. AlSabbagh, Tubular surfaces of finite type Gauss map, J. Geom. Graph. 25 (2021), pp. 45-52.

[9] B.-Y. Chen, Some open problems and conjectures on submanifolds of finite type, Soochow J. Math. 17 (1991), pp. 169-188.

[10] B.-Y. Chen, Total mean curvature and submanifolds of finite type. Second edition, World Scientific Publisher, (2014).

[11] B.-Y. Chen, A report on submanifolds of _finite type, Soochow J. Math. 22 (1996), pp. 117-337. 
[12] B.-Y. Chen, Finite type submanifolds and generalizations, University of Roma (1985).

[13]F. Dilen, J. Pas, L. Verstraelen, On surfaces of finite type in Euclidean space, Kodai Math. J. 13 (1990), pp. 10-21.

[14]F. Dillen, J. Pas, and L. Verstralen, On the Gauss map of surfaces of revolution, Bull. Inst. Math. Acad. Sinica 18 (1990), no. 3, pp. 239-246.

[15] H. Huck, U. Simon, R. Roitzsch, W. Vortisch, R. Walden, B. Wegner, and W. Wendl, Beweismethoden der Di_erentialgeometrie im Grossen, Lecture Notes in Mathematics. Vol. 335 (1973).

[16] Y. H. Kim, C. W. Lee, and D. W. Yoon, On the Gauss map of surfaces of revolution without parabolic points, Bull. Korean Math. Soc. 46 (2009), No. 6, pp. 11411149.

[17]B. Senoussi, H. Al-Zoubi, Translation surfaces of finite type in $\mathrm{Sol}_{3}$, Comment. Math. Univ. Carolin. 61 (2020), pp. 237-256.

[18] S. Stamatakis, H. Al-Zoubi, On surfaces of finite Chentype, Results. Math., 43 (2003), pp. 181-190.

[19]S. Stamatakis, H. Al-Zoubi, Surfaces of revolution satisfying $\Delta^{I I I} \boldsymbol{x}=\mathrm{A} \boldsymbol{x}$, J. for Geom. and Graphics, 14 (2010), pp. 181-186.

Hassan Al-Zoubi was born on 11-2-1970 in Irbid, Jordan. He joined the Aristotle University of Thessaloniki in 1989 and graduated in 1995. After graduation he studied for one year the German language and one year later he started his Ph.D studies and in 2001 he was awarded a Ph. D in Mathematics and he began his research on differential geometry.

In 2004 started his work as an assistant prof. in AlZaytoonah University of Jordan in Amman capital of Jordan till now. His publications:

1. Introduction to Geometry, dar alam althaqafa, 2014.

Some published articles:

1. On surfaces of finite Chen type. 2003, Result. Math. 43: 181-190.

2. Surfaces of revolutions satisfying $\Delta^{\mathrm{III}} \mathbf{x}=\Lambda \mathbf{x}$, Journal for Geometry and Graphics 2010.

3. Ruled and Quadric surfaces satisfying $\Delta^{I I I} \boldsymbol{x}=\mathrm{A} \boldsymbol{x}$. Journal for Geometry and Graphics, 20 (2016) 147157.

\section{Creative Commons Attribution License 4.0 (Attribution 4.0 International, CC BY 4.0)}

This article is published under the terms of the Creative Commons Attribution License 4.0

https://creativecommons.org/licenses/by/4.0/deed.en_US 